\title{
Clinicopathological analysis of CD8-positive lymphocytes in the tumor parenchyma and stroma of hepatocellular carcinoma
}

\author{
JUN-LING AN ${ }^{1}$, QIAO-HONG JI ${ }^{1}$, JI-JIANG AN ${ }^{2}$, SHINJI MASUDA ${ }^{3}$ and KOICHI TSUNEYAMA ${ }^{4}$ \\ ${ }^{1}$ Department of Pathology and Pathophysiology, Medical College, Henan University of Science and Technology, \\ Luoyang, Henan 471003; ${ }^{2}$ The Emergency Department, Songxian People's Hospital, Luoyang, Henan 471400, P.R. China; \\ ${ }^{3}$ Department of Diagnostic Pathology, Koseiren Takaoka Hospital, Toyama 933-0335; ${ }^{4}$ Department of Diagnostic Pathology, \\ Graduate School of Medicine and Pharmaceutical Sciences, University of Toyama, Toyama 930-0194, Japan
}

Received December 30, 2013; Accepted July 15, 2014

DOI: $10.3892 / 01.2014 .2516$

\begin{abstract}
Tumor-infiltrating lymphocytes may be a manifestation of antitumor immunity. In the present study, hepatocellular carcinoma (HCC) and pericancerous non-tumor liver tissues samples were obtained from 86 surgical patients who had not received preoperative treatment. The cellular expression levels of CD4 and CD8 were immunohistochemically examined in the two tissue groups using tissue microarrays, to evaluate their clinicopathological relevance. Immunohistochemically, CD4 and CD8 T cells were observed in the tumor parenchyma and tumor stroma, and the intensity of CD4 and CD8 immunoreactivity was homogeneous in all HCC samples examined. Morphometrically, the average numbers of CD4and CD8-positive $\mathrm{T}$ cells were significantly increased in the tumor stroma, compared with those in the tumor parenchyma (tumor stroma versus tumor parenchyma: $22 \pm 3.6$ versus $7.4 \pm 0.9$ in $\mathrm{CD} 4,32.8 \pm 4.2$ versus $16 \pm 2.5$ in $\mathrm{CD} 8$; both $\mathrm{P}<0.01$ ). Furthermore, the average numbers of CD8-positive T cells in the tumor parenchyma and stroma were significantly increased, compared with the average numbers of CD4-positive cells $(\mathrm{P}<0.05)$. In addition, in the tumor parenchyma and stroma, the average numbers of CD8 $\mathrm{T}$ cells were significantly higher in patients with tumor diameters $\leq 5 \mathrm{~cm}$ compared with those in patients with tumor diameters $>5 \mathrm{~cm}$ (diameter $\leq 5 \mathrm{~cm}$ versus diameter $>5 \mathrm{~cm}: 18.1 \pm 3.3$ versus $12.2 \pm 3.8$ in tumor parenchyma, $36.5 \pm 4.8$ versus $21.9 \pm 8.9$ in tumor stroma; both $\mathrm{P}<0.05)$. In addition, CD8 expression was significantly enhanced in patients with chronic hepatitis and cirrhosis, compared with paired tumor parenchymal tissues $(\mathrm{P}<0.01)$. Furthermore, a significant positive correlation was observed
\end{abstract}

Correspondence to: Professor Koichi Tsuneyama, Department of Diagnostic Pathology, Graduate School of Medicine and Pharmaceutical Sciences, University of Toyama, 2630 Sugitani, Toyama 930-0194, Japan

E-mail:ktsune@med.u-toyama.ac.jp

Key words: hepatocellular carcinoma, tumor parenchyma, tumor stroma, CD4 T cells, CD8 T cells between CD4 and CD8 expression in the tumor parenchyma and stroma (both $\mathrm{P}<0.001$ ). These observations suggest that tumor parenchyma- or stroma-infiltrating CD8 T cells may be involved in HCC tumor diameter control.

\section{Introduction}

Hepatocellular carcinoma (HCC) is the fifth most common type of cancer, with $>600,000$ HCC cases developing annually worldwide (1). HCC commonly occurs in patients secondary to chronic hepatitis or cirrhosis resulting from either hepatitis $\mathrm{B}$ or $\mathrm{C}$ virus infection, or from non-virus-related causes, such as alcohol or aflatoxin exposure (2-4). A persistent, non-specific and ineffective immune system activation within the chronically inflamed liver is hypothesized to induce carcinogenesis $(2,3,5)$. Current treatments for HCC include surgical resection, liver transplantation and local ablative therapies, such as percutaneous ethanol injection, thermal ablation and intra-arterial chemoembolization (6). However, $>75 \%$ patients relapse within five years and the overall survival for HCC patients remains poor $(7,8)$. Therefore, the development of more effective therapeutic tools and strategies is required.

A number of studies have suggested that the tumor microenvironment is important in tumor development, tumor control and the response to treatment (9-14). In breast, colorectal and lung cancer, as well as HCC, the status of the stroma and the local adaptive immune response are superior prognostic factors compared with tumor phenotype or clinical staging (11-14). In clinicopathological practice, intratumoral infiltration of CD4 or CD8 T cells was found to be correlated with lower disease recurrence and improved survival rates in HCC (14-16) and ovarian carcinoma (17). Furthermore, HCC tumor size was also found to have prognostic significance $(7,18,19)$. In human colorectal tumors, the type, density and location of tumor-infiltrating immune cells have been reported to be predictors of clinical outcome (11). In a transgenic mice model, the adoptive transfer of CD8-positive cytotoxic T lymphocytes (CTLs) into immune-deficient mice markedly reduced tumor growth and tumor diameter (20). However, in human HCC, the association between tumor-infiltrating immune cells and tumor size is less understood. In the present study, the association between T-cell type, location and the biological behavior in human 
HCC specimens was investigated, particularly focusing on CD4 and CD8 T cells in the tumor parenchyma and stroma.

\section{Materials and methods}

HCC specimens. A total of 86 cases of HCC (61 males and 25 females) were selected from medical records at Koseiren Takaoka Hospital (Toyama, Japan). In each case, HCC was carefully diagnosed as determined by macroscopic and histopathological findings. As a control, corresponding pericancerous non-tumor liver tissues (at least $3 \mathrm{~cm}$ away from the tumor site) were also analyzed. None of the individuals had suffered metastasis or had received prior treatment, such as percutaneous ethanol injection, thermal ablation or intra-arterial chemoembolization, which may influence HCC biological behavior, prior to surgery. The HCC samples were classified into four groups according to the International Union Against Cancer tumor-node-metastasis (TNM) classification (21). Pericancerous non-tumor liver tissues were also classified into the following four groups according to the modified histological activity index system (22): Non-chronic hepatitis $(\mathrm{NCH})$, chronic hepatitis $(\mathrm{CH})$, chronic hepatitis with pre-cirrhotic changes (pre-cirrhotic stage, $\mathrm{PC}$ ) or cirrhosis (C). The detailed profiles of all HCC cases (gender, age, tumor diameter, differentiation, Edmondson staging (23), nodule number, TNM staging, and infiltration into hepatic vein, portal vein or capsule) are shown in Tables I and II. This study was approved by the ethics committee of Koseiren Takaoka Hospital (Toyama, Japan) and written informed consent was obtained from all patients.

Tissue microarray. Tissue microarrays were constructed as described previously (24). Briefly, in each case, hematoxylin and eosin-stained HCC sections and paired pericancerous liver tissue sections (designated as tumor and peritumor, respectively) were observed under a microscope (Olympus SZX10; Olympus Corporation, Tokyo, Japan). Representative areas of lymphocyte infiltration, away from the necrotic and hemorrhagic areas, were marked and punched with a cylinder ( $3 \mathrm{~mm}$ in diameter) followed by transferal to a recipient block. In total, 172 cores were punched and distributed into 11 recipient blocks. The lesions were placed in duplicate cores adjacent to one another. The blocks were then embedded in paraffin for sectioning at $4 \mu \mathrm{m}$.

Immunohistochemistry. Briefly, following deparaffinization, the sections were subjected to antigen retrieval under microwave heating with target retrieval solution (Dako Cytomation, Kyoto, Japan) for $15 \mathrm{~min}$. Thereafter, the sections were immersed in $0.3 \% \mathrm{H}_{2} \mathrm{O}_{2}$ in methanol for $30 \mathrm{~min}$ to inhibit endogenous peroxidase activity. The sections were then incubated for $15 \mathrm{~min}$ with rabbit anti-human CD4 polyclonal antibodies (1:100; Santa Cruz Biotechnology, Inc., CA, USA) and rabbit anti-human CD8 polyclonal antibodies (1:100; Santa Cruz Biotechnology, Inc.) in phosphate-buffered saline containing $1 \%$ normal goat serum (Wako Pure Chemical Industries, Ltd., Tokyo, Japan) and $1 \%$ bovine serum albumin (Wako Pure Chemical Industries, Ltd.) under intermittent microwave irradiation, as previously described $(25,26)$. Envision ${ }^{+}$(Dako Cytomation) for rabbit immunoglobulin was added and the sections were incubated under intermittent microwave irradiation for $15 \mathrm{~min}$. Positive reactions were visualized with 3,3'-diaminobenzidine tetrahydrochloride.

Morphometrical analysis. CD4- and CD8-positive lymphocytes were classified into the following three groups according to cell distribution: Tumor parenchyma lymphocytes, which were located within a cancer cell nest; tumor stroma lymphocytes, with lymphocytes located in the stroma contacting the cancer cells; and peritumor parenchyma lymphocytes, which were located in the pericancerous liver parenchyma. Morphometrical analysis was performed according to methods described in a previous study (27), for semi-quantitative evaluation of the immunohistochemical findings by two investigators without prior knowledge. Briefly, in each case, using an Olympus SZX10 microscope (Olympus Corporation), 15 independent and intact high power microscopic areas (magnification, $\mathrm{x} 400$ ) with the most abundant lymphocyte infiltrations were selected (five tumor parenchyma, five tumor stroma and five peritumor parenchyma areas), and the numbers of CD4 and CD8 T cells were counted in each microscopic field. The average numbers of CD4 and CD8 T cells in the five selected microscopic fields signified the CD4 and CD8 expression levels in each HCC or pericancerous liver tissue specimen. For the evaluation of CD4 and CD8 immunoreactions in the tumor stroma, 35 cases were omitted since distinguishing the carcinoma stroma from the surrounding carcinoma parenchyma in these cases was difficult.

Statistical analysis. The mean and standard error of the mean were calculated for all parameters determined in this study. Statistical analysis was performed using the nonparametric Mann-Whitney U test, one-factor analysis of variance or Spearman's correlation coefficient by rank test. $\mathrm{P}<0.05$ was considered to indicate a statistically significant difference.

\section{Results}

Lymphocyte distribution. In the HCC samples, CD4 and CD8 T cells were observed in the tumor parenchyma and tumor stroma (Fig. 1A and B), and the intensity of CD4 or CD8 immunoreactivity was homogeneous in all samples examined. The numbers of CD4- and CD8-positive T cells appeared fewer in the tumor parenchyma, compared with those in tumor stroma. In order to semi-quantitatively evaluate the immunohistochemical findings, morphometrical analysis was performed. As shown in Fig. 1C, the average numbers of CD4-and CD8-positive T cells were significantly increased in the tumor stroma, compared with those in the tumor parenchyma (tumor stroma versus tumor parenchyma: $\mathrm{CD} 4,22 \pm 3.6$ versus $7.4 \pm 0.9$; $\mathrm{CD} 8,32.8 \pm 4.2$ versus $16 \pm 2.5$; both $\mathrm{P}<0.01$ ). Furthermore, the average numbers of CD8-positive T cells in tumor parenchyma and tumor stroma were significantly increased, compared with the numbers of CD4-positive cells (CD8 versus CD4: tumor parenchyma, $16 \pm 2.5$ versus $7.4 \pm 0.9, \mathrm{P}<0.01$; tumor stroma, $32.8 \pm 4.2$ versus $22 \pm 3.6, \mathrm{P}<0.05)$. This observation suggests that CD8 $\mathrm{T}$ cells were predominant in the host anticancer cellular immunity.

Association between CD4 and CD8 expression and HCC behavior. In the tumor parenchyma and stroma, no significant differences in the CD4 immunoreactions were observed 
Table I. Association between the numbers of CD4- and CD8-positive lymphocytes in hepatocellular carcinoma tumor parenchymal tissues, and patient clinicopathological characteristics.

\begin{tabular}{|c|c|c|c|c|c|}
\hline $\begin{array}{l}\text { Clinicopathological } \\
\text { characteristic }\end{array}$ & No. & $\begin{array}{l}\text { No. CD4 T cells, } \\
\text { mean } \pm \text { SEM }\end{array}$ & P-value(s) & $\begin{array}{l}\text { No. CD8 T cells, } \\
\text { mean } \pm \text { SEM }\end{array}$ & P-value(s) \\
\hline \multicolumn{6}{|c|}{ Age at diagnosis, years } \\
\hline$\leq 60$ & 19 & $7.2 \pm 1.7$ & 0.623 & $15.2 \pm 4.3$ & 0.911 \\
\hline$>60$ & 67 & $7.6 \pm 1.7$ & & $16.5 \pm 3.0$ & \\
\hline \multicolumn{6}{|l|}{ Gender } \\
\hline Male & 61 & $7.0 \pm 0.9$ & 0.137 & $16.1 \pm 2.6$ & 0.061 \\
\hline Female & 25 & $6.4 \pm 1.8$ & & $15.8 \pm 5.9$ & \\
\hline \multicolumn{6}{|l|}{ Tumor diameter, $\mathrm{cm}$} \\
\hline$\leq 5$ & 56 & $7.8 \pm 1.3$ & 0.604 & $18.1 \pm 3.3$ & 0.037 \\
\hline$>5$ & 30 & $6.2 \pm 1.3$ & & $12.2 \pm 3.8$ & \\
\hline \multicolumn{6}{|l|}{ Differentiation status } \\
\hline \multirow[t]{2}{*}{ Well } & 24 & $7.2 \pm 1.7$ & 0.982 & $11.0 \pm 2.3$ & 0.784 \\
\hline & & & 0.996 & & 0.255 \\
\hline \multirow[t]{2}{*}{ Moderately } & 39 & $7.6 \pm 1.5$ & 0.982 & $15.1 \pm 2.6$ & 0.784 \\
\hline & & & 0.957 & & 0.507 \\
\hline \multirow[t]{2}{*}{ Poorly } & 23 & $6.9 \pm 1.2$ & 0.996 & $22.1 \pm 7.7$ & 0.255 \\
\hline & & & 0.957 & & 0.507 \\
\hline \multicolumn{6}{|l|}{ Edmondson stage } \\
\hline I-II & 70 & $7.2 \pm 0.9$ & 0.310 & $16.4 \pm 3.3$ & 0.225 \\
\hline III-IV & 16 & $10.5 \pm 3.0$ & & $14.0 \pm 3.6$ & \\
\hline \multicolumn{6}{|l|}{ Background } \\
\hline \multirow[t]{3}{*}{$\mathrm{NCH}$} & 6 & $6.4 \pm 2.8$ & 0.998 & $24.5 \pm 16.1$ & 0.821 \\
\hline & & & 1.000 & & 0.934 \\
\hline & & & 0.978 & & 0.751 \\
\hline \multirow[t]{3}{*}{$\mathrm{CH}$} & 47 & $7.1 \pm 1.4$ & 0.998 & $15.0 \pm 3.3$ & 0.821 \\
\hline & & & 0.999 & & 0.996 \\
\hline & & & 0.980 & & 0.989 \\
\hline \multirow[t]{3}{*}{$\mathrm{PC}$} & 11 & $7.0 \pm 1.9$ & 1.000 & $16.9 \pm 6.2$ & 0.934 \\
\hline & & & 0.999 & & 0.996 \\
\hline & & & 0.991 & & 0.973 \\
\hline \multirow[t]{3}{*}{$\mathrm{C}$} & 22 & $8.1 \pm 1.8$ & 0.978 & $12.9 \pm 4.1$ & 0.751 \\
\hline & & & 0.980 & & 0.989 \\
\hline & & & 0.991 & & 0.973 \\
\hline \multicolumn{6}{|l|}{ Nodule number } \\
\hline Single & 77 & $7.4 \pm 0.9$ & 0.400 & $16.7 \pm 2.7$ & 0.667 \\
\hline Double & 9 & $6.6 \pm 2.8$ & & $9.9 \pm 3.0$ & \\
\hline \multicolumn{6}{|c|}{ Portal vein infiltration } \\
\hline Yes & 17 & $6.2 \pm 2.6$ & 0.667 & $21.7 \pm 7.8$ & 0.633 \\
\hline No & 69 & $7.3 \pm 0.9$ & & $14.5 \pm 2.4$ & \\
\hline \multicolumn{6}{|l|}{ Hepatic vein invasion } \\
\hline Yes & 16 & $7.2 \pm 1.9$ & 0.709 & $13.8 \pm 4.1$ & 0.228 \\
\hline No & 70 & $7.4 \pm 1.0$ & & $16.7 \pm 3.0$ & \\
\hline \multicolumn{6}{|c|}{ Infiltration into capsule } \\
\hline Yes & 29 & $7.4 \pm 1.4$ & 0.608 & $17.7 \pm 5.2$ & 0.812 \\
\hline No & 57 & $7.2 \pm 1.1$ & & $15.1 \pm 2.7$ & \\
\hline \multicolumn{6}{|l|}{ TNM stage } \\
\hline I-II & 54 & $7.7 \pm 1.2$ & 0.364 & $16.3 \pm 3.1$ & 0.301 \\
\hline III-IV & 32 & $6.0 \pm 1.1$ & & $13.2 \pm 3.7$ & \\
\hline
\end{tabular}

P-values were obtained with nonparametric Mann-Whitney U test or one-factor analysis of variance. Bold P-value denotes statistical significance. SEM, standard error of the mean; $\mathrm{NCH}$, non-chronic hepatitis; $\mathrm{CH}$, chronic hepatitis; $\mathrm{PC}$, chronic hepatitis with cirrhotic changes; $\mathrm{C}$, cirrhosis. 
Table II. Association between the numbers of CD4- and CD8-positive lymphocytes in hepatocellular carcinoma tumor stromal tissues, and patient clinicopathological characteristics.

\begin{tabular}{|c|c|c|c|c|c|}
\hline $\begin{array}{l}\text { Clinicopathological } \\
\text { characteristic }\end{array}$ & No. & $\begin{array}{l}\text { CD4 T cells, } \mathrm{n}, \\
\text { mean } \pm \mathrm{SEM}\end{array}$ & P-value(s) & $\begin{array}{l}\text { CD8 T cells, } \mathrm{n}, \\
\text { mean } \pm \mathrm{SEM}\end{array}$ & P-value(s) \\
\hline \multicolumn{6}{|c|}{ Age at diagnosis, years } \\
\hline$\leq 60$ & 12 & $23.9 \pm 10.6$ & 0.400 & $33.3 \pm 10.0$ & 0.842 \\
\hline$>60$ & 39 & $21.8 \pm 3.8$ & & $33.1 \pm 4.8$ & \\
\hline \multicolumn{6}{|l|}{ Gender } \\
\hline Male & 37 & $24.0 \pm 4.6$ & 0.315 & $31.3 \pm 4.1$ & 0.849 \\
\hline Female & 14 & $17.2 \pm 5.9$ & & $37.8 \pm 11.5$ & \\
\hline \multicolumn{6}{|l|}{ Tumor diameter, $\mathrm{cm}$} \\
\hline$\leq 5$ & 40 & $23.1 \pm 4.4$ & 0.519 & $36.5 \pm 4.8$ & 0.022 \\
\hline$>5$ & 11 & $19.4 \pm 7.2$ & & $21.9 \pm 8.9$ & \\
\hline \multicolumn{6}{|l|}{ Differentiation status } \\
\hline \multirow[t]{2}{*}{ Well } & 10 & $20.7 \pm 6.8$ & 0.911 & $28.3 \pm 5.9$ & 0.870 \\
\hline & & & 0.971 & & 0.886 \\
\hline \multirow[t]{2}{*}{ Moderately } & 27 & $24.7 \pm 6.0$ & 0.911 & $34.0 \pm 6.1$ & 0.870 \\
\hline & & & 0.757 & & 0.999 \\
\hline \multirow[t]{2}{*}{ Poorly } & 14 & $18.1 \pm 5.1$ & 0.971 & $34.3 \pm 9.5$ & 0.886 \\
\hline & & & 0.757 & & 0.999 \\
\hline \multicolumn{6}{|l|}{ Edmondson stage } \\
\hline I-II & 31 & $20.7 \pm 4.0$ & 0.317 & $32.7 \pm 4.4$ & 0.858 \\
\hline III-IV & 20 & $27.4 \pm 8.6$ & & $36.7 \pm 11.5$ & \\
\hline \multicolumn{6}{|l|}{ Background } \\
\hline \multirow[t]{3}{*}{$\mathrm{NCH}$} & 3 & $8.8 \pm 5.5$ & 0.894 & $5.3 \pm 3.9$ & 0.530 \\
\hline & & & 0.154 & & 0.149 \\
\hline & & & 0.946 & & 0.665 \\
\hline \multirow[t]{3}{*}{$\mathrm{CH}$} & 33 & $20.9 \pm 4.4$ & 0.894 & $32.1 \pm 5.5$ & 0.530 \\
\hline & & & 0.117 & & 0.416 \\
\hline & & & 0.997 & & 0.996 \\
\hline \multirow[t]{3}{*}{$\mathrm{PC}$} & 4 & $54.9 \pm 25.3$ & 0.154 & $59.0 \pm 19.9$ & 0.149 \\
\hline & & & 0.117 & & 0.416 \\
\hline & & & 0.141 & & 0.424 \\
\hline \multirow[t]{3}{*}{$\mathrm{C}$} & 11 & $18.9 \pm 5.7$ & 0.946 & $29.7 \pm 6.8$ & 0.665 \\
\hline & & & 0.997 & & 0.996 \\
\hline & & & 0.141 & & 0.424 \\
\hline \multicolumn{6}{|l|}{ Nodule number } \\
\hline Single & 43 & $22.2 \pm 4.1$ & 0.728 & $32.7 \pm 4.8$ & 0.368 \\
\hline Double & 8 & $21.6 \pm 6.9$ & & $36.9 \pm 7.6$ & \\
\hline \multicolumn{6}{|l|}{ Portal vein infiltration } \\
\hline Yes & 9 & $24.1 \pm 8.0$ & 0.652 & $43.2 \pm 16.4$ & 0.942 \\
\hline No & 42 & $22.0 \pm 4.1$ & & $31.3 \pm 3.8$ & \\
\hline \multicolumn{6}{|l|}{ Hepatic vein invasion } \\
\hline Yes & 15 & $22.5 \pm 6.2$ & 0.879 & $42.0 \pm 10.5$ & 0.558 \\
\hline No & 36 & $22.5 \pm 4.6$ & & $31.2 \pm 4.1$ & \\
\hline \multicolumn{6}{|c|}{ Infiltration into capsule } \\
\hline Yes & 18 & $21.9 \pm 5.0$ & 0.573 & $38.2 \pm 9.0$ & 0.700 \\
\hline No & 33 & $22.3 \pm 4.9$ & & $29.0 \pm 3.5$ & \\
\hline \multicolumn{6}{|l|}{ TNM stage } \\
\hline I-II & 31 & $23.9 \pm 5.3$ & 0.978 & $31.8 \pm 4.9$ & 0.867 \\
\hline III-IV & 20 & $20.3 \pm 4.7$ & & $34.7 \pm 7.7$ & \\
\hline
\end{tabular}

P-values were obtained with nonparametric Mann-Whitney U test or one-factor analysis of variance. Bold P-value denotes statistical significance. SEM, standard error of the mean; $\mathrm{NCH}$, non-chronic hepatitis; $\mathrm{CH}$, chronic hepatitis; $\mathrm{PC}$, chronic hepatitis with cirrhotic changes; $\mathrm{C}$, cirrhosis. 


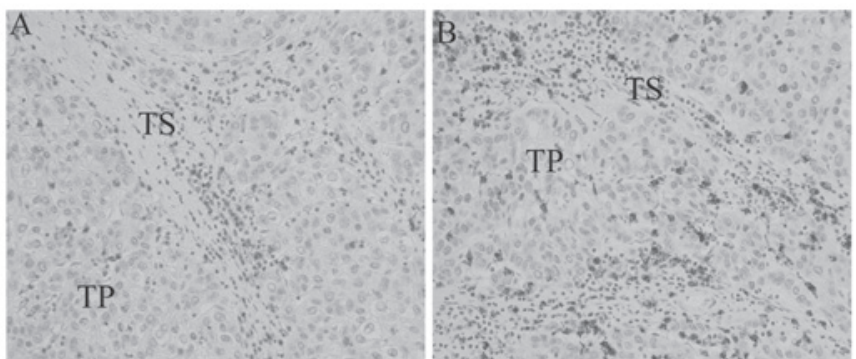

$\mathrm{C}$

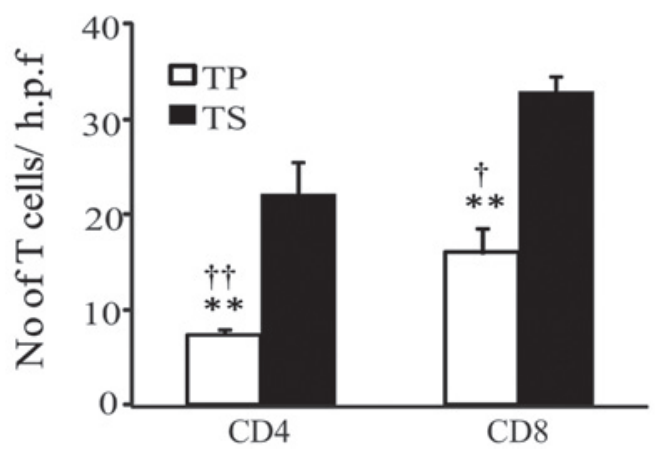

Figure 1. Immunohistochemical analysis of T-cell expression in the TP and TS of hepatocellular carcinoma samples (original magnification, $\mathrm{x} 400$ ). (A) $\mathrm{CD}^{+}$and (B) $\mathrm{CD} 8^{+} \mathrm{T}$-cell expression in TP and TS. (C) Morphometrical analysis was performed. Data are presented as the mean \pm standard error of the mean. ${ }^{* *} \mathrm{P}<0.01$, TP vs. TS in CD4 and CD8; ${ }^{\dagger} \mathrm{P}<0.01, \mathrm{CD} 4$ vs. CD8 in $\mathrm{TP}$; ${ }^{\dagger}<0.05, \mathrm{CD} 4$ vs. CD8 in TS. Statistical analyses were performed using the Mann-Whitney U test. TP, tumor parenchyma; TS, tumor stroma; h.p.f., high-power field.

between patients with tumor diameters $\leq 5 \mathrm{~cm}$ and patients with tumor diameters $>5 \mathrm{~cm}$ (both P>0.05; Tables I and II). By contrast, the average numbers of CD8 T cells in the tumor parenchyma and tumor stroma were significantly increased in patients with tumor diameters $\leq 5 \mathrm{~cm}$ compared with patients with tumor diameters $>5 \mathrm{~cm}$ (diameter $\leq 5 \mathrm{~cm}$ versus diameter $>5 \mathrm{~cm}$ : tumor parenchyma, $18.1 \pm 3.3$ versus $12.2 \pm 3.8$; tumor stroma, $36.5 \pm 4.8$ versus 21.9 \pm 8.9 ; both $\mathrm{P}<0.05$; Tables I and II; Fig. 2A-C). Furthermore, in the tumor parenchyma and stroma, no significant differences in either CD4 or CD8 immunoreactivity were detected between age, gender, differentiation, Edmondson staging (23), liver disease background, number of nodules, TNM stage or infiltration into the portal vein, hepatic vein or the capsule variables (Tables I and II). These observations suggest that the numbers of CD8 T cells in HCC parenchyma and stroma may not be correlated with tumor progression or metastasis, but may be correlated with tumor volume.

Association between CD4 and CD8 expression and background hepatic disease. As shown in Fig. 3, in the $\mathrm{CH}$ and $\mathrm{C}$ background groups, CD8 expression levels in the peritumor parenchymas were significantly higher than those in the paired tumor parenchymas (peritumor parenchyma vs. tumor parenchyma: $\mathrm{CH}$ background, $18.4 \pm 1.4$ vs. 15.0 \pm 3.3 ; C background, $19.8 \pm 2.2$ vs. $12.9 \pm 4.1$; both $\mathrm{P}<0.01)$. By contrast, in the $\mathrm{NCH}$ and $\mathrm{PC}$ background groups, no significant differences in CD8 expression were detected between the tumor parenchyma and peritumor parenchyma (Fig. 3). Furthermore, in HCC and pericancerous liver tissues from all background groups, no significant differences in the CD4 $\mathrm{T}$ cells between the

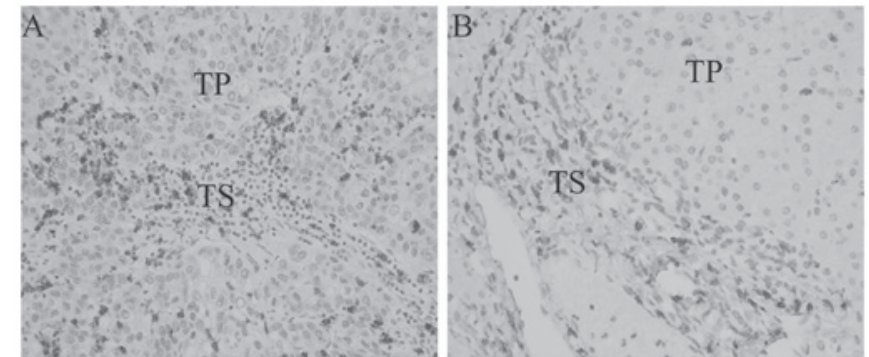

$\mathrm{C}$

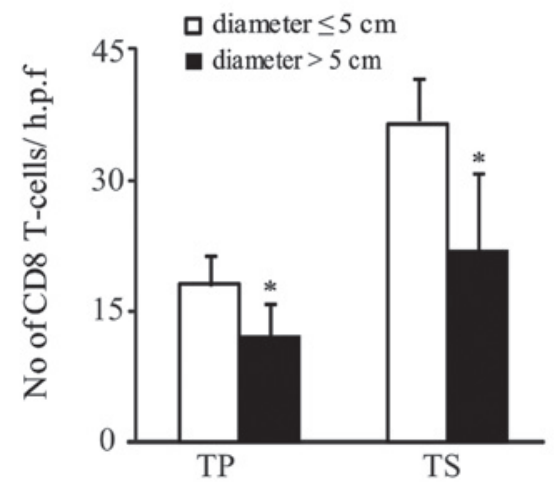

Figure 2. Immunohistochemical analysis of $\mathrm{CD}^{+} \mathrm{T}$-cell expression in the $\mathrm{TP}$ and TS of hepatocellular carcinoma samples (original magnification, $\mathrm{x} 400$ ). $\mathrm{CD}^{+}$T-cell expression in patients with tumor diameters $(\mathrm{A}) \leq 5 \mathrm{~cm}$ and (B) $>5 \mathrm{~cm}$. (C) Morphometrical analysis was performed. Data are presented as the mean \pm standard error of the mean. ${ }^{*} \mathrm{P}<0.05$, diameter $\leq 5 \mathrm{~cm}$ group vs. diameter $>5 \mathrm{~cm}$ group in TP and TS. TP, tumor parenchyma; TS, tumor stroma; h.p.f., high-power field.

tumor parenchyma and peritumor parenchyma (peritumor parenchyma versus tumor parenchyma: $\mathrm{NCH}$ background, 4.1 \pm 1.4 vs. $6.4 \pm 2.8 ; \mathrm{CH}$ background, $7.9 \pm 1.8$ vs. $7.1 \pm 1.4$; PC background, $10.0 \pm 3.3$ vs. $7.0 \pm 1.9$; cirrhosis background, $7.6 \pm 2.5$ vs $8.1 \pm 1.8$, all $\mathrm{P}>0.05$ ) were identified.

Correlation between CD4 and CD8 expression in HCC. Spearman's correlation analysis revealed that CD8 expression was positively correlated with CD4 expression in the tumor parenchyma and tumor stroma (correlation coefficient $=0.62$ in tumor parenchyma; correlation coefficient $=0.68$ in tumor stroma; both $\mathrm{P}<0.001$; Fig. $4 \mathrm{~A}$ and B).

\section{Discussion}

Solid tumors are composed of parenchyma (neoplastic cells) and stroma. Neoplastic cells are also usually dispersed within the stroma, which is composed of fibroblasts, endothelial cells and a variety of immune cells $(28,29)$. These stromal cells are key in tumor development, tumor control and the response to treatment (9-14). In the present study, the distribution of tumor-infiltrating lymphocytes (TIL) within the tumor parenchyma or tumor stroma was investigated in order to more accurately evaluate the respective impacts of these TILs on the biological behavior of HCC. To the best of our knowledge, no studies have been conducted with regard to the TIL expression in different areas of tumors in association with HCC clinicopathological parameters. The results of the present study revealed significant differences in the intratumoral expression of CD8, but not CD4. 


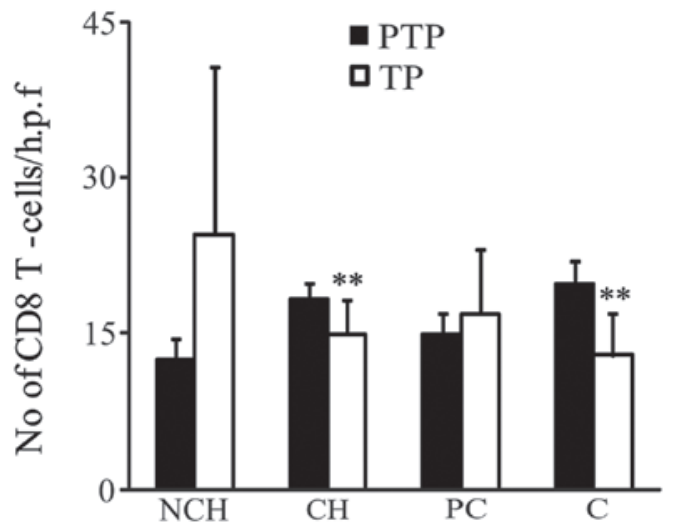

Figure 3. CD8 expression in hepatocellular carcinoma (TP) and paired peritumor liver tissues (PTP). Morphometrical analysis was performed for semi-quantitative evaluation of the immunohistochemical findings. Data are presented as the mean \pm standard error of the mean. Statistical analysis was performed using the nonparametric Mann-Whitney U test. ${ }^{* *} \mathrm{P}<0.01$, PTP vs. TP in $\mathrm{CH}$ and $\mathrm{C}$ background groups. $\mathrm{NCH}$, non-chronic hepatitis; $\mathrm{CH}$, chronic hepatitis; $\mathrm{PC}$, pre-cirrhotic stage; $\mathrm{C}$, cirrhosis; PTP, peritumor parenchyma; TP, tumor parenchyma; h.p.f., high-power field.

In the present study, a difference in the number of CD8 $\mathrm{T}$ cells between the tumor parenchyma and stroma in HCC (tumor parenchyma $<$ tumor stroma) was detected. This may be explained by the evidence from a previous study that tumor microenvironments are rich in immune-cell-derived chemokines (10).

CD8 T cells exert a central role in the immune defense against cancer. For example, CD8-positive CTLs directly contact and kill tumor cells by releasing membrane-lytic granules, such as perforin and granzyme. Indeed, the presence of tumor antigen-specific CD8 T cells has been observed in HCC patients (30). CD8-positive CTLs also kill tumor stroma cells that cross-present antigens. In addition, CTL-derived cytokines, including tumor necrosis factor $\alpha$, interleukin 4 (IL-4) and IL-10, contribute to tumor rejection by inhibition of tumor stroma formation (20,31-33). In the present study, the average numbers of CD8 T cells in the tumor parenchyma and stroma were higher in patients with tumor diameters $\leq 5 \mathrm{~cm}$ than in patients with tumor diameters $>5 \mathrm{~cm}$. In concurrence with this finding, Gao et al (16) demonstrated that primary tumor size was inversely correlated with the presence of CD8 $\mathrm{T}$ cells in $\mathrm{HCC}$, although no distinction was made regarding the precise location of the T cells. Additionally, in the center (CT) and the invasive margin (IM) of colorectal cancer tumors, CD3, CD8, GZMB (a marker for CD8-positive CTLs) and CD45RO (a marker for memory $\mathrm{T}$ cells) expression levels in each tumor region (CT and IM) were negatively correlated with tumor recurrence. High CD8 density, and CD45RO and GZMB expression were correlated with longer overall survival times (11). A study conducted by Chew et al (14) further confirmed and complemented these findings; NK and $\mathrm{CD}^{+} \mathrm{T}$ cells were observed to be the main proliferating lymphocytes in human HCC. The presence of NK and CD8 ${ }^{+}$ $\mathrm{T}$ cells was associated with longer survival times, which is concurrent with the finding from another previous study that host anticancer cellular immunity is mainly attributable to CD8-positive CTLs (15). Collectively, these observations suggest that an increased number of CD8 T cells in HCC is
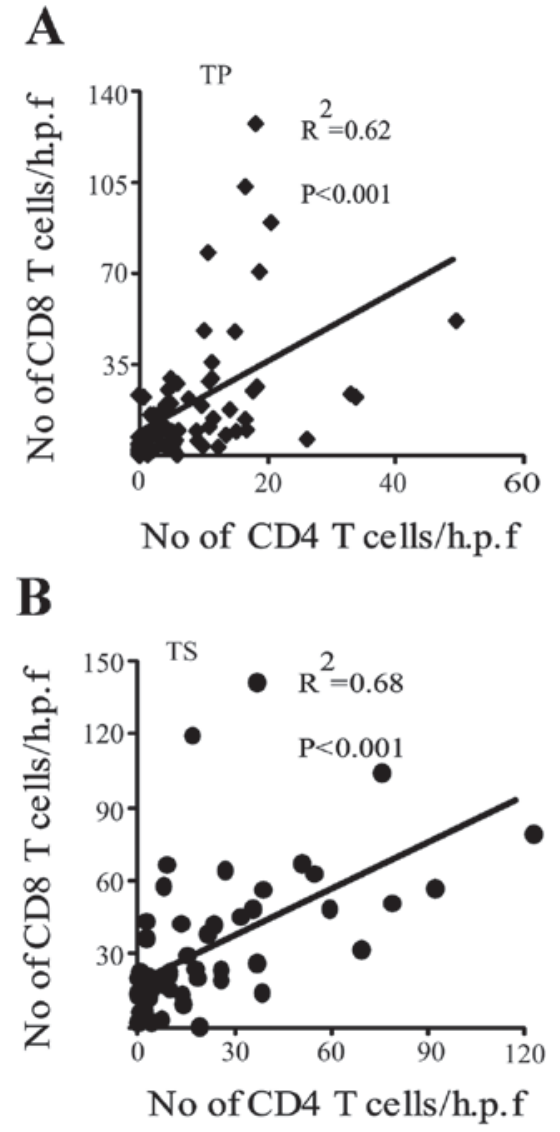

Figure 4. Correlation between CD4 and CD8 expression in (A) TP and (B) TS of HCC samples. Statistical analyses were performed using Spearman's correlation coefficient by rank test. $\mathrm{P}<0.001$. TP, tumor parenchyma; TS, tumor stroma; HCC, hepatocellular carcinoma; h.p.f., high-power field.

associated with longer overall survival times and improved prognosis.

Another finding in the present study was that CD8 expression was significantly increased in the peritumor chronic hepatitis and cirrhotic parenchymas, compared with those in paired tumor parenchymas. This finding is concurrent with the results of a study revealing that the proportion of immune-suppressed regulatory $\mathrm{T}$ cells was significantly higher in HCC than that in the non-tumorous liver (34).

The results from the present study demonstrate that CD8-positive T cells are not only important in tumor size control but may also be a valuable prognostic factor. However, the present study did not take account of factors such as survival analysis, phenotypic characterizations (naïve, activated or regulated) and cytotoxic function. Therefore, further studies are required, particularly those that use human $\mathrm{HCC}$ specimens with known survival times following $\mathrm{HCC}$ resection.

The present study demonstrated that elevated CD8 expression in tumor parenchyma and tumor stroma was correlated with reduced tumor diameter. Therefore, tumor parenchyma and tumor stroma infiltrating CD8 T cells were shown to be involved in HCC diameter control.

\section{Acknowledgements}

The authors would like to thank Mr. Tokimasa Kumada and Mr. Hideki Hatta for aid and technical assistance. 


\section{References}

1. Schütte K, Bornschein J and Malfertheiner P: Hepatocellular carcinoma - epidemiological trends and risk factors. Dig Dis 27: 80-92, 2009.

2. Kremsdorf D, Soussan P, Paterlini-Brechot $\mathrm{P}$ and Brechot $\mathrm{C}$ : Hepatitis B virus-related hepatocellular carcinoma: paradigms for viral-related human carcinogenesis. Oncogene 25: 3823-3833, 2006.

3. Nakamoto Y, Guidotti LG, Kuhlen CV, Fowler P and Chisari FV: Immune pathogenesis of hepatocellular carcinoma. J Exp Med 188: 341-350, 1998.

4. Fattovich G and Llovet JM: Risk factors for hepatocellular carcinoma in HCV-cirrhosis: what we know and what is missing. J Hepatol 44: 1013-1016, 2006.

5. Naugler WE, Sakurai T, Kim S, et al: Gender disparity in liver cancer due to sex differences in MyD88-dependent IL-6 production. Science 317: 121-124, 2007.

6. Parmiani $\mathrm{G}$ and Anichini A: T cell infiltration and prognosis in HCC patients. J Hepatol 45: 178-181, 2006.

7. Tobe T, Uchino J, Endo Y, Oto M, Okamoto E, Kojiro M, et al: Predictive factors for long term prognosis after partial hepatectomy for patients with hepatocellular carcinoma in Japan. The Liver Cancer Study Group of Japan. Cancer 74: 2772-2780, 1994.

8. Levy I and Sherman M; Liver Cancer Study Group of the University of Toronto: Staging of hepatocellular carcinoma: assessment of the CLIP, Okuda, and Child-Pugh staging systems in a cohort of 257 patients in Toronto. Gut 50: 881-885, 2002 .

9. Balkwill $\mathrm{F}$ and Mantovani A: Inflammation and cancer: back to Virchow? Lancet 357: 539-545, 2001.

10. de Visser KE, Eichten A and Coussens LM: Paradoxical roles of the immune system during cancer development. Nat Rev Cancer 6: 24-37, 2006.

11. Galon J, Costes A, Sanchez-Cabo F, et al: Type, density, and location of immune cells within human colorectal tumors predict clinical outcome. Science 313: 1960-1964, 2006.

12. Dieu-Nosjean MC, Antoine M, Danel C, et al: Long-term survival for patients with non-small-cell lung cancer with intratumoral lymphoid structures. J Clin Oncol 26: 4410-4417, 2008.

13. Finak G, Bertos N, Pepin F, et al: Stromal gene expression predicts clinical outcome in breast cancer. Nat Med 14: 518-527, 2008.

14. Chew V, Tow C, Teo M, et al: Inflammatory tumour microenvironment is associated with superior survival in hepatocellular carcinoma patients. J Hepatol 52: 370-379, 2010.

15. Wada Y, Nakashima O, Kutami R, Yamamoto O and Kojiro M: Clinicopathological study on hepatocellular carcinoma with lymphocytic infiltration. Hepatology 27: 407-414, 1998.

16. Gao Q, Qiu SJ, Fan J, et al: Intratumoral balance of regulatory and cytotoxic T cells is associated with prognosis of hepatocellular carcinoma after resection. J Clin Oncol 25: 2586-2593, 2007.

17. Zhang L, Conejo-Garcia JR, Katsaros D, et al: Intratumoral $\mathrm{T}$ cells, recurrence, and survival in epithelial ovarian cancer. N Engl J Med 348: 203-213, 2003.

18. Kashef E and Roberts JP: Transplantation for hepatocellular carcinoma. Semin Oncol 28: 497-502, 2001.
19. Lu XY, Xi T, Lau WY, et al: Pathobiological features of small hepatocellular carcinoma: correlation between tumor size and biological behavior. J Cancer Res Clin Oncol 137: 567-575, 2011.

20. Zhang B, Zhang Y, Bowerman NA, et al: Equilibrium between host and cancer caused by effector T cells killing tumor stroma. Cancer Res 68: 1563-1571, 2008.

21. The general rules for the clinical and pathological study of primary liver cancer. Liver Cancer Study Group of Japan. Jpn J Surg 19: 98-129, 1989.

22. Ishak K, Baptista A, Bianchi L, et al: Histological grading and staging of chronic hepatitis. J Hepatol 22: 696-699, 1995.

23. Edmondson HA and Steiner PE: Primary carcinoma of the liver: a study of 100 cases among 48,900 necropsies. Cancer 7: 462-503, 1954

24. Kononen J, Bubendorf L, Kallioniemi A, et al: Tissue microarrays for high-throughput molecular profiling of tumor specimens. Nat Med 4: 844-847, 1998.

25. Hatta H, Tsuneyama K, Kumada T, et al: Freshly prepared immune complexes with intermittent microwave irradiation result in rapid and high-quality immunostaining. Pathol Res Pract 202: 439-445, 2006.

26. Kumada T, Tsuneyama K, Hatta H, Ishizawa S and Takano Y: Improved 1-h rapid immunostaining method using intermittent microwave irradiation: practicability based on 5 years application in Toyama Medical and Pharmaceutical University Hospital. Mod Pathol 17: 1141-1149, 2004.

27. Sato E, Olson SH, Ahn J, et al: Intraepithelial CD8+ tumor-infiltrating lymphocytes and a high $\mathrm{CD} 8+$ /regulatory $\mathrm{T}$ cell ratio are associated with favorable prognosis in ovarian cancer. Proc Natl Acad Sci USA 102: 18538-18543, 2005.

28. Liotta LA and Kohn EC: The microenvironment of the tumour-host interface. Nature 411: 375-379, 2001.

29. Connolly JL, Schnitt SJ, Wang HH, Dvorak AM and Dvorak HF. Principles of cancer pathology. In: Holland-Frei Cancer Medicine. Bast RC Jr, Kufe DW, Pollock RE, Weichselbaum RR, Holland JF and Frei E III (eds). BC Decker, Inc., Hamiton, ON, Canada, pp384-399, 2000.

30. Zerbini A, Pilli M, Soliani P, et al: Ex vivo characterization of tumor-derived melanoma antigen encoding gene-specific CD8+cells in patients with hepatocellular carcinoma. J Hepatol 40: 102-109, 2004.

31. Spiotto MT and Schreiber H: Rapid destruction of the tumor microenvironment by CTLs recognizing cancer-specific antigens cross-presented by stromal cells. Cancer Immun 5: 8, 2005.

32. Singh S, Ross SR, Acena M, Rowley DA and Schreiber H: Stroma is critical for preventing or permitting immunological destruction of antigenic cancer cells. J Exp Med 175: 139-146, 1992.

33. Blankenstein T: The role of tumor stroma in the interaction between tumor and immune system. Curr Opin Immunol 17: $180-186,2005$

34. Kobayashi N, Hiraoka N, Yamagami W, et al: FOXP3+ regulatory $\mathrm{T}$ cells affect the development and progression of hepatocarcinogenesis. Clin Cancer Res 13: 902-911, 2007. 